\title{
Accidentes viales de los motociclistas en México: subgrupos y factores de riesgo
}

\author{
Luis David Berrones-Sanz \\ Universidad Autónoma de la Ciudad de México \\ Victoria Alejandra Muro.-Báez \\ Secretariado Técnico del Consejo Nacional para la Prevención de Accidentes, México
}

\section{RESUMEN}

En México, la motocicleta como modo de transporte ha tenido un rápido crecimiento. En la última década, aumentó $338.05 \%$ el número de estos vehículos, por lo que actualmente, representa $5.97 \%$ del parque vehicular nacional. Sin embargo, en este periodo, el promedio anual indica que $3.16 \%$ de los motociclistas sufrieron un accidente, lo que vuelve al motociclista un usuario vulnerable y de alto riesgo en la seguridad vial. El objetivo de esta investigación es identificar subgrupos de motociclistas con un riesgo particularmente alto de accidente y precisar los factores de riesgo. El estudio, de corte cuantitativo, con una muestra conformada por todas las muertes y lesiones de motociclistas reportados, entre el año 2000 y 2014, en la base de datos del Instituto Nacional de Estadística y Geografía y de la Dirección General de Información en Salud. Las variables descriptivas, se determinaron para todas las categorías y se emparejaron con la causa básica de la muerte para encontrar correlación estadística. En México, los sistemas de información mencionados, registraron para el año 2014, más de 41,881 accidentes y 826 muertes a causa de accidentes de motocicleta; con mayor frecuencia en hombres ( $\mathrm{P}<0.001)$, entre 20 y 30 años de edad, en donde $20.55 \%$ de las lesiones son en la cabeza y sólo $16.59 \%$ de los lesionados usaban casco. La combinación de edad, género y el no usar casco, parecen ser un potente factor de riesgo. Sin embargo, existe gran sub-registro en los datos, debido a que el formato de atención por violencia o lesión (SIS-SS-17-P) no incluye a la motocicleta como agente de lesión, por lo que se destaca la necesidad de crear un sistema estadístico y, sobre todo, fomentar la cultura de buenas prácticas viales, promocionar los factores protectores y equipos de seguridad

\section{INTRODUCCIÓN}

Generalmente el motociclismo es considerado una actividad peligrosa (Mannering \& Grodsky, 1995) ya que sus usuarios tienen un riesgo particularmente alto de accidentes (Huang, B., Preston, J., 2004; Chesham, Rutter, \& Quine, 1993). Aunque en otros países los accidentes en motocicleta se han reducido drásticamente (Bjornskau, Naevestad, \& Akhtar, 2011), en México, las lesiones, en estos vehículos, son un problema creciente que simplemente se atribuyen al incremento de la tasa vehicular, ya que han aumentado $338.05 \%$ su número en la última década. Las motocicletas resultan atractivas por varios factores: los 
costos asociados al vehículo y el rendimiento del combustible, las bajas emisiones contaminantes, la velocidad en ciudades cada vez más congestionadas y el prestigio. Los esfuerzos o principales preocupaciones están enfocados en reducir las lesiones, en la cabeza y en el cerebro, a través reglamentos de tránsito en la que se obliga el uso de los cascos de seguridad, pero se deja de lado los modelos de la psicología social, en la que se deben utilizar las creencias y conductas de las personas, predecir su comportamiento e influir en una conducción segura. De aquí, que este trabajo presenta un análisis de los accidentes en motocicleta con el objetivo de identificar subgrupos y características de motociclistas con un riesgo particularmente alto de accidentes.

\section{METODOLOGÍA}

Se analizan los registros de los vehículos de motor registrados en circulación y los accidentes de tránsito terrestre del Instituto Nacional de Estadística y Geografía (INEGI); junto con la base de datos de lesionados de la Dirección General de Información en Salud.

La población bajo estudio incluye el total de accidentes en motocicletas $(\mathrm{N}=41881)$ y el total de lesionados ( $\mathrm{N}=13916)$ durante el año 2014. Se utilizaron todas las variables disponibles: región anatómica de la lesión, referencia temporal, características del accidente, referencia geográfica, características del conductor responsable y del lesionado, entre otras.

El análisis estadístico incluye la descripción, categorización y asociación de variables, para lo que se utilizó pruebas de diferencia de proporciones y Chi-Square $\left(\chi^{2}\right)$. En las variables asociadas, se realizó el cálculo de la odds ratio (OR) con un intervalo de confianza del $95 \%$ (IC del 95\%), para la comparación, se utilizó principalmente el tipo de accidente (fatal, no fatal) o las lesiones en la parte anatómica de la cabeza.

\section{EL PARQUE VEHICULAR EN MÉXICO}

Para el año 2014, a nivel nacional se reportan poco más de 38 millones de vehículos de motor registrados en circulación; de éstos, $67.18 \%$ corresponden a automóviles, $0.91 \%$ a camiones para pasajeros, $25.94 \%$ para camiones y camionetas de carga y, $5.97 \%$ a motocicletas; estos últimos, tienen la proporción más alta de la historia. En la gráfica 1, se puede observar el comportamiento en el número de vehículos que han tenidos los automóviles y las motocicletas desde el año 1980, y como las motocicletas han tenido, en la última década, un crecimiento más rápido. Así, del año 2004 al 2014, la cantidad de motocicletas en el país aumentó 3.38 veces, con una tasa de crecimiento medio anual de $15.92 \%$.

Sin embargo, el país no se comporta de forma homogénea, por ejemplo: en el crecimiento absoluto, del número de motocicletas, a lo largo de las 33 entidades federativas, llama la atención, que en ésta misma década, sólo el estado de Coahuila tuvo un comportamiento decreciente $(-38.18 \%)$, del resto, tres estados aumentaron significativamente su número motocicletas: Oaxaca con $751.47 \%$, Tlaxcala con $984.98 \%$ y Estado de México con un crecimiento muy superior al promedio de $4929.01 \%$. No obstante, el Estado de México es la entidad más poblada del país y sólo se registran 1.46 motocicletas por cada cien habitantes, 
por lo que Quintana Roo con $8.99 \%$, Campeche con $7.83 \%$ y Yucatán con 5.82\%, son las entidades con más motociclistas por habitantes. Por su parte, Coahuila con $0.09 \%$ se mantiene con la menor proporción de motocicletas por habitantes.

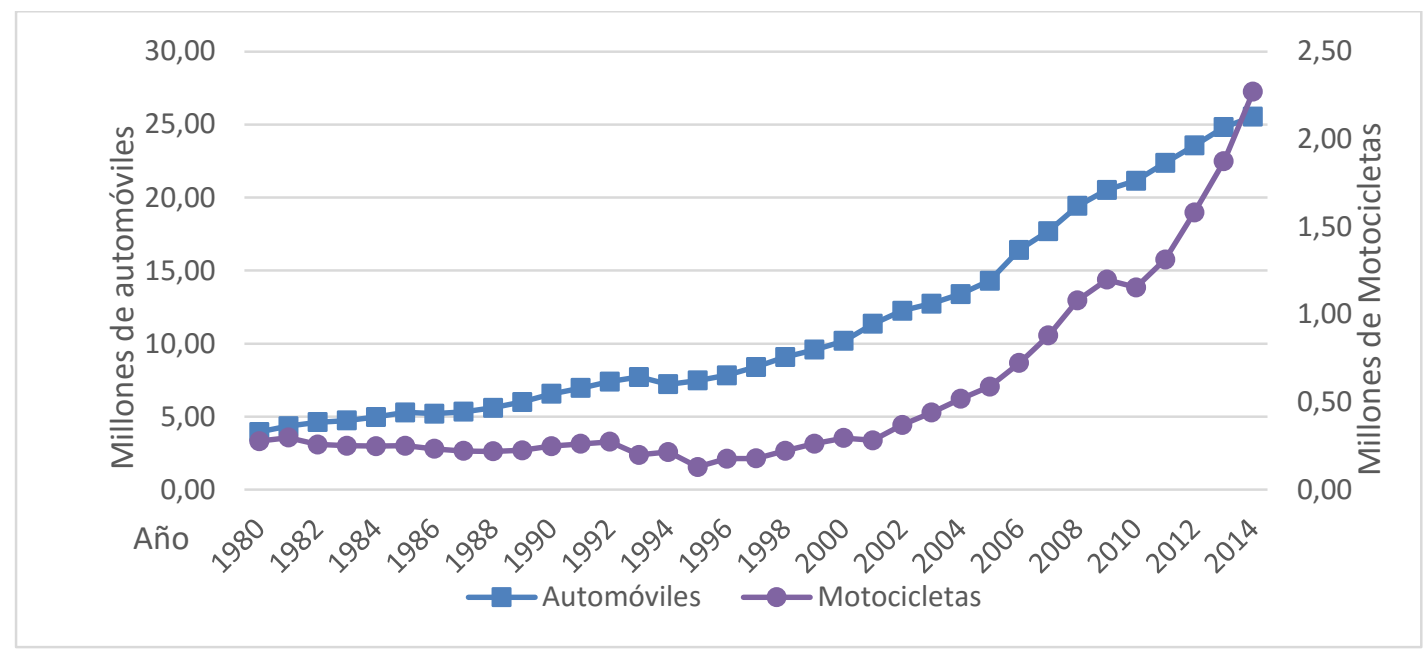

Gráfica 1 - Evolución del parque vehicular en México.

\section{LOS ACCIDENTES DE MOTOCICLETAS EN MÉXICO}

Para el año 2014, el Instituto Nacional de Estadística y Geografía (INEGI) registra, en el territorio nacional, 41,881 accidentes en motocicleta, por lo que se tienen 34.98 (95\% CI: $34.6,35.3)$ accidentes por cada cien mil habitantes. Como se puede observar en la gráfica 2 , los accidentes tienen una tendencia creciente y, en la última década, tuvieron un crecimiento absoluto de $96 \%$ a un ritmo promedio de $6.95 \%$ por año. Obviamente, el aumento en el parque vehicular de las motocicletas se correlaciona positivamente con el número de accidentes $(\mathrm{R}=0.93, \mathrm{P}<0.001)$ y, lamentablemente, con el número de muertos; cuya tasa, cercana a 2.5 muertos cada millón de habitantes, paso a una relación de 6.9 por millón (95\% IC: $6.4,7.4)$. Además, las proporciones son más críticas, al relacionar los accidentes con el número de vehículos, ya que en el 2014, 1.84\% (95\% IC: 1.83, 1.86) de las motocicletas se vieron involucradas en un accidente y, murieron 3.64 (95\% IC: $3.39,3.89$ ) personas por cada diez mil motocicletas.

Las cifras, también aumentan para los lesionados, que representan $57.20 \%$ de los accidentes e imponen una tasa de 20 lesionados cada cien mil habitantes, (95\% IC: 19.75, 20.26) y de 1055/100000 (95\% IC: 1041.73, 1068.31) para los lesionados por motocicleta registrada.

La figura 1 muestra un mapa temático (graduated colors) de la cantidad total de accidentes por entidad federativa, las gráficas de pastel muestran la proporción de motos accidentadas en las motos registradas, y los puntos muestran la cantidad de motocicletas registradas en cada estado. 


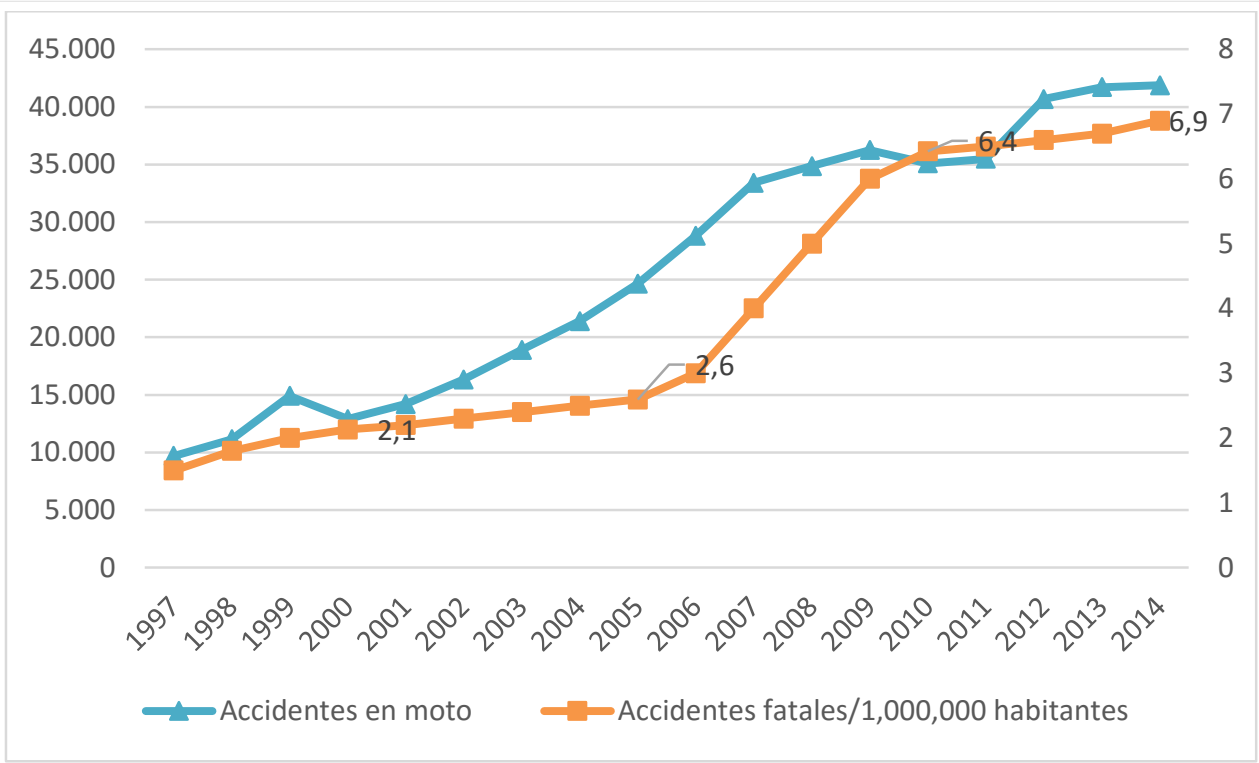

Gráfica 2 - Accidentes viales en México.

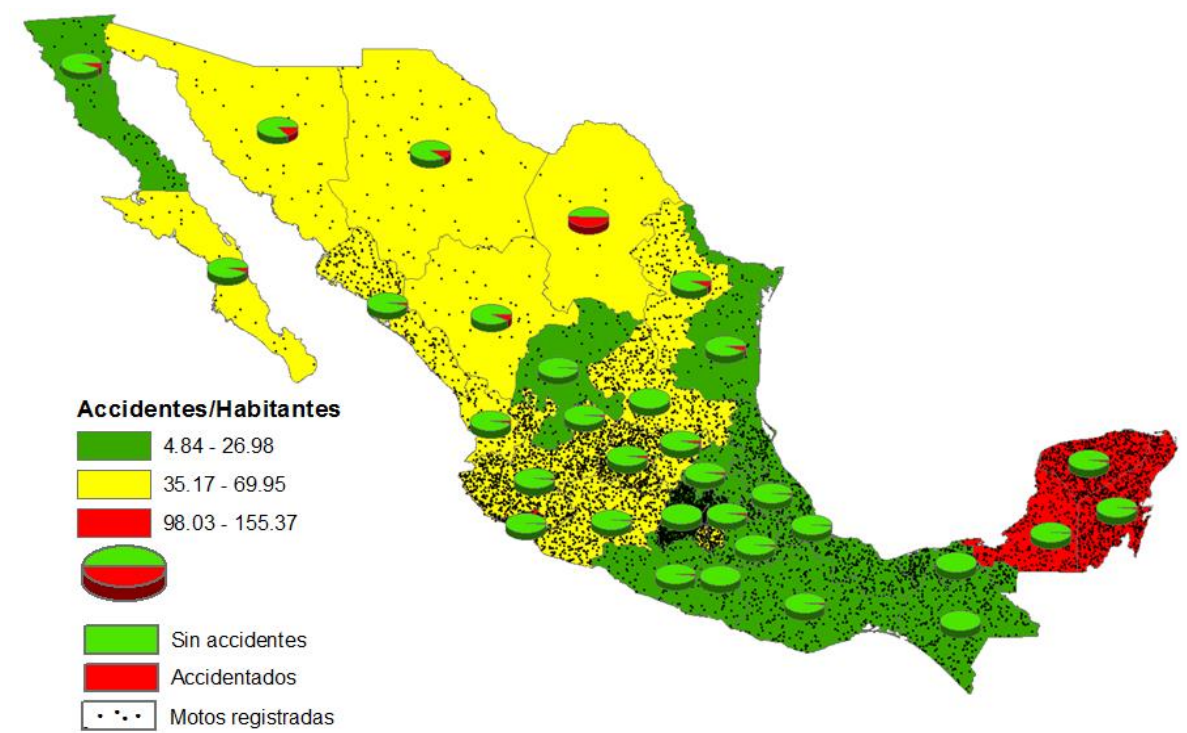

Fig. 1 - Mapa de accidentes en México.

El sureste del país - Campeche, Quintana Roo y Yucatán- con la mayor tasa de motocicletas por habitante) coinciden con el mayor número de accidentes por habitantes -respectivamente 108.71, 155.37 y 124.74 muertos por cada cien mil habitantes-, además, Quintana Roo, es el estado con mayor tasa de mortalidad, 29.41 muertos por cada millón de habitantes, seguido por, Sinaloa 24.67/1 000000 y Aguascalientes 18.11/1000 000. Resulta interesante, que en el norte del país, a pesar de la baja tasa de motorización y de que los accidentes por habitantes se sitúan en valores medios, los accidentes por número de motocicletas registradas se incrementan. Así, en los Charts Pie, se puede observar, por ejemplo: a Coahuila con 56.75 muertos por cada diez mil motocicletas registradas, a Sonora con 22.13/10 000 y a Chihuahua con 13.14/10 000. Por el contrario, el Estado de México, a pesar del valor atípico (outlier) que ha registrado, en el incremento que de motocicletas, aparece con los valores más bajos de accidentes en todos los indicadores 
Tanto el número de accidentes por habitantes (4.84/100 000) como en el número de muertos por motocicletas registradas $(0.53 / 10000)$.

\section{CARACTERÍSTICAS SOCIODEMOGRÁFICAS DE LOS LESIONADOS EN ACCIDENTES DE MOTOCICLETAS EN MÉXICO}

En los lesionados por accidente de motocicleta en México, se ubican al género masculino, con $76.6 \%$ de prevalencia, en estos, $49.3 \%$ son menores a 25 años y $60.1 \%$ tienen hasta estudios de secundaria; por lo que el género, representa la principal diferencia entre las características analizadas. Sin embargo, el 30.2\% de los lesionados no son conductores, son acompañantes, y en esta categoría, las mujeres son las más afectadas, ya que aquí, se sitúa $53.74 \%$ del total de las mujeres, la mayor proporción para este género.

\begin{tabular}{|c|c|c|c|c|c|}
\hline Categoría & Variable & Fatal & No fatal & Solo daños & P-Value \\
\hline \multirow[t]{3}{*}{ Sexo conductor } & Se fugó & 162 & 2,995 & 1,722 & $\mathrm{P}<0.001$ \\
\hline & Hombre & 628 & 18,401 & 13,647 & \\
\hline & Mujer & 36 & 2,558 & 1,732 & \\
\hline \multirow[t]{5}{*}{ Causa del accidente } & Conductor & 804 & 22,788 & 16,397 & $\mathrm{P}<0.001$ \\
\hline & Peatón o pasajero & 9 & 390 & 21 & \\
\hline & Falla del vehículo & 4 & 90 & 64 & \\
\hline & Mala condición del camino & 4 & 359 & 396 & \\
\hline & Otra & 5 & 327 & 223 & \\
\hline \multirow[t]{2}{*}{ Superficie rodamiento } & Pavimentada & 779 & 23,527 & 16,937 & $\mathrm{P}<0.001$ \\
\hline & No pavimentada & 47 & 427 & 164 & \\
\hline \multirow[t]{2}{*}{ Zona ocurrió accidente } & Zona urbana & 486 & 21,652 & 16,290 & $\mathrm{P}<0.001$ \\
\hline & Zona suburbana & 340 & 2,302 & 811 & \\
\hline \multirow[t]{3}{*}{ Cond aliento alcohólico } & $\mathrm{Si}$ & 74 & 1,744 & 737 & $\mathrm{P}<0.001$ \\
\hline & No & 338 & 14,920 & 10,247 & \\
\hline & Se ignora & 414 & 7,290 & 6,117 & \\
\hline \multirow[t]{8}{*}{ Edad accidentado } & $12-20$ & 132 & 3768 & 2701 & $\mathrm{P}<0.001$ \\
\hline & $21-30$ & 223 & 6530 & 4724 & \\
\hline & $31-40$ & 111 & 4314 & 3253 & \\
\hline & $41-50$ & 70 & 2653 & 1955 & \\
\hline & $51-60$ & 35 & 1439 & 1039 & \\
\hline & $61-90$ & 11 & 932 & 576 & \\
\hline & No especificado & 82 & 1,323 & 1,131 & \\
\hline & No aplica porque el conductor se fugó & 162 & 2995 & 1722 & \\
\hline \multirow[t]{7}{*}{ Día de la semana } & Lunes & 108 & 3,359 & 2,407 & $\mathrm{P}<0.001$ \\
\hline & Martes & 74 & 3,077 & 2,312 & \\
\hline & Miércoles & 92 & 3,181 & 2,422 & \\
\hline & Jueves & 98 & 3,027 & 2,368 & \\
\hline & Viernes & 88 & 3,560 & 2,719 & \\
\hline & Sábado & 172 & 4,113 & 2,709 & \\
\hline & Domingo & 194 & 3,637 & 2,164 & \\
\hline \multirow[t]{12}{*}{ Tipo de accidente } & Colisión con vehículo automotor & 29 & 1,535 & & $\mathrm{P}<0.001$ \\
\hline & Colisión con peatón (atropellamiento) & 11 & 107 & 30 & \\
\hline & Colisión con animal & 119 & 838 & 522 & \\
\hline & Colisión con objeto fijo & 51 & 851 & 200 & \\
\hline & Volcadura & 14 & 599 & & \\
\hline & Caída de pasajero & 59 & 456 & 162 & \\
\hline & Salida del camino & & 7 & 4 & \\
\hline & Incendio & 1 & 4 & 4 & \\
\hline & Colisión con ferrocarril & 461 & 16,861 & 14,558 & \\
\hline & Colisión con motocicleta & 12 & 369 & 128 & \\
\hline & Colisión con ciclista & 21 & 765 & 281 & \\
\hline & Otro & & & & \\
\hline
\end{tabular}

Tabla 1 - Accidentes viales en México según clase de accidente.

En los lesionados, se reporta que $13.73 \%$ se encontraba bajo efectos del alcohol, $0.30 \%$ bajo efectos de drogas ilegales y $0.17 \%$ que había consumido drogas médicas. En la región 
anatómica de la lesión, 71.42\% de las defunciones son atribuidas a afecciones en la cabeza, sin embargo, dentro de las lesiones, las áreas con mayor incidencia son las catalogadas como múltiples (24.56\%), cabeza y cara (22.86\%) extremidades inferiores (20.61\%) y extremidades superiores (12.21\%). Ninguno de los fallecidos tenia equipo de seguridad; en general, sólo $24.6 \%$ de los lesionados traía casco u otro equipo de seguridad, de estos, $16.01 \%$ de los lesionados en la cabeza portaba casco y $36.32 \%$ de los lesionados en otras regiones anatómicas traían casco.

Dentro de la temporalidad, el mes de agosto presento la mayor cantidad de accidentes $(9.73 \%)$ y enero la menor (6.09\%), principalmente los fines de semana, sábados (16.81\%) y domingos $(21.53 \%)$. Otros resultados se muestran en la tabla 1.

\section{DISCUSIÓN}

Es bien sabido que los motociclistas son un grupo particularmente vulnerable en la carretera, algunos estudios indican que el riesgo de sufrir lesiones en motocicleta es entre 10 y 30 veces superior al correspondiente de los conductores de automóviles (Bjornskau, Naevestad, \& Akhtar, 2011; Bjornskau, Naevestad, \& Akhtar, 2011; Elvik, Vaa, \& Monclús, 2006); esto, es debido no sólo por la forma del vehículo, que mantiene los cuerpos al intemperie y limita, en comparación con los automóviles, las opciones en los sistemas de seguridad pasiva -como el cinturón de seguridad o la misma cabina del vehículo-, sino que además de la falta de protección contra choques, están expuestos a no ser vistos por otros conductores de automóviles y, a los impactos con barreras de seguridad, que han sido diseñadas para otro tipo de vehículos.

A nivel nacional, los datos del INEGI indican que las motocicletas contribuyen con 5.97\% del total de vehículos de motor registrados en circulación, son responsables del $5.84 \%$ de los accidentes viales y del $13.14 \%$ de los fallecidos por esta causa de muerte. Sin embargo, en México, el número de automóviles es 11.25 veces mayor que las motocicletas y, no obstante, que las comparaciones, en términos absolutos, subestiman la gravedad de los accidentes y la vulnerabilidad de ciertos grupos -que se muestran mejor en términos relativos-, ya que tanto el número de vehículos, como el de viajes y kilómetros, hacen que el número de accidentes, no sean directamente comparables. A pesar de esto, de los resultados obtenidos, se puede observar que los accidentes en motocicleta, tienen una proporción de 0.09 (1:10.78), en comparación con los accidentes en automóvil; proporción que cambia a 1.04 (1:0.96), al considerar, en lugar de la población, la razón de accidentes con el número de vehículos y, a 1:3.5, al considerar sólo los accidentes fatales.

Respecto a la parte del cuerpo lesionada, la cabeza es la más importante, incluso en las heridas múltiples, las heridas en la cabeza y los traumatismos intracraneales tienen una fuerte presencia. A pesar de los esfuerzos legislativos por normar el uso del casco, la cultura del mexicano es un fuerte componente en las lesiones, ya que paradójicamente, es común observar que el casco se utiliza sólo para cumplir con el reglamente de tránsito y se utiliza de tamaño o forma inadecuada, por ejemplo: niños con casco de adulto, o personas que portaban el casco en el brazo. La intención es evitar la sanción y no proteger las lesiones. El 
reciente reglamento de tránsito, publicado en el año 2015, ya se incorpora, en el artículo 37, que el casco debe ir colocado en la cabeza, sin embargo, en México, no existe norma que regule los cascos, ni en la utilización ni en la comercialización, y es frecuente ver cascos muy baratos, pero inadecuados y fuera de normas internacionales. Sin embargo, crear normas que garanticen la eficiencia del casco, es un tema polémico, principalmente por porque los casco sometidos a estándares Semell y DOT (en USA) o ECE/ONU R22 en Europa, tienen costos que en ocasiones superan hasta $10 \%$ del valor de una escooter o motocicleta debajo cilindraje; si sumamos chamarras, guates, botas y otros equipos protectores, resulta más costoso, el equipo de seguridad, que las motocicletas que más se comercializan en este país. Esto representa un serio problema debido al bajo poder adquisitivo de la población, por lo que la regulación sobre el uso y comercialización del casco representan medidas no populistas, sobre todo porque la motocicleta ha surgido para suplir, las necesidades de movilidad, en un sistema de transporte público que es incómodo y poco atractivo para la población, pero también, porque a pesar de que la seguridad no es negociable, algunos individuos promueven el derecho de los motociclistas a movilizarse bajo su propio riesgo, con el argumento de que no afectan a terceros.

Elvik (2013) indica que un motociclista reduce, con el uso del casco, 25\% la probabilidad de resultar lesionado, $30 \%$ si utiliza otro equipo protector (como ropa de cuero) por lo que en conjunto, con todo el equipo se reduce cerca de 50\%; por su parte, Hurt et al. (1981) establece que motociclistas con casco mostraron significativamente menor lesión en la cabeza y el cuello, en todos los niveles de severidad, para todo tipo de lesiones. De cualquier forma, para México, es clara la necesidad de crear campañas de sensibilización, ya que a pesar de que los reglamentos de tránsito, en algunas ciudades, tienen más de una década, de haber incorporado el uso del casco como obligatorio, los resultados indican que $83.99 \%$ de los lesionados no portaban ningún tipo de casco y, esta conducta de riesgo, expone a los motociclistas a 2.11 veces mayor probabilidad de sufrir lesiones en la cabeza respecto a los que si utilizan el casco (OR=2.11, IC 95\%; 1.67, 2.17). Si bien, la cifra de prevalencia del uso del casco, parece ser inferior $(16.07 \%$ vs $78 \%, \mathrm{P}<0.001)$ a datos de investigaciones previas (Hidalgo-Solórzano, Inclán-Valadez, Pérez-Núñez, \& Híjar, 2012), el estudio de comparación no incluye lesionados, sino puros motociclistas en circulación; sin embargo, existen datos en los que al comparar las proporciones parece no haber diferencia, por ejemplo: dentro de los que usan el casco, la mayoría (83.29\%) son conductores y, el resto, son pasajeros (16.71\%); en el estudio de comparación, en esta variable se registra $84 \%$, por lo que al realizar la prueba de hipótesis para diferencia de proporciones no se puede rechazar que sean diferentes ( 83.29 vs $84 \%, \mathrm{P}=0.520$ ), lo mismo ocurre al comparar el género de las personas que utilizaban casco, donde los hombres predominan $(78.48 \% \mathrm{Vs} 81 \% \mathrm{P}=0.026)$. Por su parte, Otte et al. (1984) refieren que $72.5 \%$ de los lesionados no usaban casco y, de estos, $70 \%$ sufrieron lesiones en la cabeza, mientras que para los que los que si usaron, $45 \%$ presentaron lesión en la cabeza. Estos datos difieren con los resultados del estudio, donde sólo $18.52 \%$ de los que no usaron casco, sufrieron lesión en la cabeza y $9.70 \%$ entre los que usaron casco, que sufrieron lesión en esta zona anatómica.

En otros factores, que se observan en los lesionados, están los referentes a la vía y la zona 
geográfica, donde a pesar de que $98.48 \%$ de los accidentes ocurren en superficies pavimentadas, es 3.3 veces más probable un accidente fatal en superficies no pavimentadas (95\%IC: $2.33,4.09)$ y 6.5 veces más probable en zona suburbana (95\% IC: $5.13,6.69)$. El riesgo de accidente fatal es 4.33 veces más probable durante el día, que durante la noche, lo que se puede explicar por la gran cantidad de tránsito, a pesar de que en la noche se alcanzan mayores velocidades y de que los motociclistas son menos visibles, las vías son menos transitadas. Elliot et al. (2003), indica que en la Gran Bretaña, alrededor de 60\% de los lesionados y $40 \%$ de los fallecidos en motocicleta, implica la colisión con un coche, y alrededor de $20 \%$ y $25 \%$, de lesionados y fallecidos, son accidentes de un solo vehículo. Proporciones similares a los resultados encontrados, ya que en México, 50\% de las víctimas de la motocicleta, implica la colisión con automóvil, camioneta u otros accidentes de transporte y $42.93 \%$ son accidentes sin colisión.

Los días con mayor riesgo son los sábados y domingos, con 19.13 veces más probabilidad de accidente (95\% IC: 13.22, 25.35), que el resto de la semana; en Elliot et al (2003) se argumenta, que este pico del fin de semana, es debido a los paseos recreativos, sobre todo en motocicletas con motores superiores a los $500 \mathrm{cc}$, aunque este dato no se tiene disponible, se puede inferir que en México es coincidente, ya que los fines de semana, disminuye la utilización de las motocicletas de trabajo, generalmente con cilindrajes menores.

En los conductores responsables, mientras que el consumo de alcohol incrementa 1.87 veces el riesgo (95\% IC: 1.43, 2.35), la mayoría (78.02\%) son de sexo masculino, con 2.4 veces mayor probabilidad (95\% IC: 1.70, 3.31); esto apoya, la creencia de que las mujeres, tienen tasas más bajas de participación de los accidentes, en particular entre los jóvenes pilotos. Tales diferencias de género, se han encontrado en los análisis de accidentes de numerosas investigaciones sobre motociclistas (Mannering, 1993). Sin embargo, la prevalencia de consumo de alcohol, de $13.73 \%$, es mucho menor que las encontradas por (Preusser, Williams, \& Ulmer, 1995) quien indica que entre $41 \%$ y $80 \%$ de los conductores de motocicleta lesionados, dieron positivo al consumo de alcohol.

\section{CONCLUSIONES}

A pesar de la importancia de los resultados, es necesario considerar que algunas variables de interés, no se incluyeron en este estudio por falta de información, por ejemplo: en la base de datos del INEGI, no incorpora equipo de seguridad ni región anatómica de la lesión, además de que existe gran sub-registro en los datos, debido a que el formato de atención por violencia o lesión (SIS-SS-17-P) no incluye a la motocicleta como agente de lesión. Por tal motivo, se destaca la necesidad de crear un sistema estadístico, que incluya a los conductores de motocicleta. Pese a las limitaciones de información, los resultados de este estudio, forman una aproximación al problema de los motociclistas en México y a las características que se deben incluir en los programas de acción, para prevenir sus lesiones. Es evidente que México, ha tenido un avance en materia de seguridad vial, por lo menos, desde hace varios años, se reconoce como un problema de salud y se han creado iniciativas, organismos y convenios para combatir este problema. El no uso del casco, fue uno de los seis factores de 
riesgo incluidos en el programa de la Iniciativa Mexicana de Seguridad Vial y, el uso del casco, se ha ido reforzando en la reglamentación del tránsito; además, de que ya se planean normas para comercialización de los cascos. Sin embargo, se deben crear campañas y fomentar la cultura de buenas prácticas viales, promocionar los factores protectores y equipos de seguridad en la motocicleta, mejorar la infraestructura vial y que sea adecuada para este tipo de vehículos, y utilizar la psicología social, para aumentar el porcentaje de personas que utilizan el casco y equipo protector, además, de sensibilizar a los conductores, sobre los riesgos de conducir una motocicleta, por ejemplo: en su uso recreativo, los fines de semana y bajo los efectos del alcohol.

\section{AGRADECIMIENTOS}

A la Universidad Autónoma de la Ciudad de México (UACM) que ha facilitado la realización de este trabajo.

\section{REFERENCIAS}

Bjornskau, T., Naevestad, T.-O., \& Akhtar, J. (2011). Traffic safety among motorcyclists in Norway: a study of subgroups and risk factors. Accident Analysis and Prevention, 49, págs. 50-57.

Brandt, M.-M., Ahrns, K. S., Corpron, C. A., Franklin, G. A., \& Wahl, W. L. (2002). Hospital Cost Is Reduced by Motorcycle Helmet Use. The Journal of Trauma: Injury, Infection, and Critical Care, 53(3), págs. 469-471.

Chesham, D. J., Rutter, D. R., \& Quine, L. (1993). Motorcycling safety research: A review of the social and behavioural literature. Social Science \& Medicine, 37(3), págs. 419429.

Elliott, MA,Baughan, CJ,Broughton, J,Chinn, B,Grayson, GB,Knowles, J,Smith, LR,Simpson, H. (2003). Motorcycle safety: A scoping study. Transport Research Laboratory.

Elvik, R., Vaa, T., \& Monclús, J. (2006). El manual de medidas de seguridad vial. [España]: Fundación Instituto Tecnológico para Seguridad del Automóvil.

Hidalgo-Solórzano, E., Inclán-Valadez, C., Pérez-Núñez, R., \& Híjar, M. (2012). Motorcycle non-standard helmet use in an urban area of Mexico. Injury Prevention, 18(Suppl 1), págs. A192-A192.

Huang, B., Preston, J. (2004). A Literature Review on Motorcycle Collisions. Transport Studies Unit.

Hurt, H. H., Ouellet, J. V., \& Thom, D. R. (1981). Motorcycle accident cause factors and identification of countermeasures. Los Angeles, California: Department of Transportation NHTSA.

Mannering, F. L. (1993). Male/female driver characteristics and accident risk: Some new evidence. Accident Analysis \& Prevention, 25(1), págs. 77-84.

Mannering, F. L., \& Grodsky, L. L. (1995). Statistical analysis of motorcyclists' perceived accident risk. Accident Analysis \& Prevention, 27(1), págs. 21-31.

Otte, D., Jessl, P., \& Suren, E. G. (1984). Impact points and resultant injuries to the head of 
motorcyclists involved in accidents, with and without crash helmets. The Netherlands.

Preusser, D. F., Williams, A. F., \& Ulmer, R. G. (1995). Analysis of fatal motorcycle crashes: crash typing. Accident; analysis and prevention, 27(6), págs. 845-851. 